\title{
Avaliação econômico-financeira da microgeração de energia elétrica proveniente de biogás da suinocultura
}

\author{
Angelica B. Avaci', Samuel N. M. de Souza ${ }^{2}$, Luiz I. Chaves ${ }^{2}$, \\ Carlos E. C. Nogueira ${ }^{3}$, Rosana K. Niedzialkoski ${ }^{4} \&$ Deonir Secco ${ }^{3}$
}

\begin{abstract}
RESUMO
Uma das maiores fontes de energia disponíveis nas áreas rurais e agroindustriais é a biomassa, que aparece na forma de resíduos vegetais e animais, tais como resto de colheita, esterco animal, plantações energéticas e efluentes agroindustriais. Esses resíduos podem ser utilizados pelo produtor rural ou na agroindústria para a queima direta, visando à produção de calor ou à produção de biogás em biodigestores. A produção de suínos gera grande quantidade de dejetos causando problemas ao meio ambiente, que quando não tratados possuem quantidade relevante de metano. Objetivou-se com este trabalho avaliar, economicamente, a produção de energia elétrica via biogás da suinocultura. Constatou-se que a melhor opção para o produtor é a venda de crédito carbono quando o custo de produção de energia elétrica é viável quando vendido à concessionária de energia. A melhor condição encontrada foi a de geração de $20 \mathrm{~h} \mathrm{~d}^{-1}$, caso o tempo de retorno do investimento seja de 8 anos e o valor de produção de energia elétrica, de R\$120,11 kWh.
\end{abstract}

Palavras-chave: suínos, eletricidade, biomassa

\section{Economic evaluation of microgeneration of electricity from biogas of swine manure}

\begin{abstract}
One of the biggest sources of energy available in rural areas is biomass and agribusiness. The same appears in the form of plant and animal residues such as crop residues, animal manure, energy crops and agroindustrial effluents. These residues can be used by the farmer or agribusiness for direct burning in order to produce heat or production of biogas. Swine production generates large amounts of wastes causing environmental problems if left untreated. The same has lot of methane. The objective of this study was to evaluate the production cost of electricity through biogas from swine manure. It was found that the best option for the producer is selling carbon credit when the production cost of electricity is viable when sold to utility power. The best condition was found to generate $20 \mathrm{~h} \mathrm{~d}^{-1}$, when the time for return on investment is 8 years and the production of electricity is $\mathrm{R} \$ 120.11 \mathrm{kWh}$.
\end{abstract}

Key words: swine, electricity, biomass

\footnotetext{
1 UNIOESTE. R. Universitária, 2069, Jardim Universitário, CEP 85819-110, Cascavel, PR. Fone: (45) 9994-6651. E-mail: angelicaavaci@hotmail.com

2 UNIOESTE. Fone: (45) 3220-3155. E-mail: melegsouza@hotmail.com; I_chaves@hotmail.com

${ }^{3}$ UNIOESTE. Fone: (45) 3220-3151. E-mail: cecn1@yahoo.com.br; deonir.secco@unioeste.br

${ }^{4}$ UNIOESTE. Fone: (45) 9962-8332. E-mail: rkrauss86@gmail.com
} 


\section{INTRODUÇÃO}

Para as próximas décadas há perspectivas de crise no setor energético, devidoao descompasso entre o crescimento da demanda e a incapacidade da oferta acompanhar o ritmo de expansão do Produto Interno Bruto (PIB) mundial, em especial do petróleo, que é base da matriz energética nacional. $\mathrm{O}$ resultado imediato e perceptível de tal crise se refere à volatilidade e aos recordes no preço do barril no petróleo. Países buscam aliviar as incertezas e, de alguma forma, impedir que suas economias caiam diante da crise energética, estimulando as fontes energéticas renováveis, como biomassa solar e eólica.

O Brasil é um país que apresenta tradição no uso de fontes renováveis de energia, destacando-se o uso da energia hidrelétrica como responsável pela maior parcela, isto é, $74 \%$ de toda a geração de eletricidade. Por outro lado, existem ainda estáticos, com pouco aproveitamento, um enorme potencial de fontes renováveis de energia ressaltando-se, entre elas, a energia eólica $(0,4 \%)$ e a biomassa $(4,7 \%)$ (BEN, 2011).

Com o aumento dos valores das fontes convencionais de energia juntamente com a crescente preocupação do futuro da oferta de energia, a segurança energética ganhou posição significativa nos meios políticos, ao redor do mundo, incluindo o Brasil. Reconhecido pelas fontes renováveis de energia, o Brasil tornou-se centro da atenção de países desenvolvidos em virtude da sua fonte de energia limpa de bicombustíveis e geração de energia elétrica.

Conforme dados disponibilizados pelo IBGE (2006) o Brasil é o quarto produtor mundial de suínos contando com cerca de 43,2 milhões de cabeças, apresentando-se com grande potencial de resíduos. Os resíduos rurais incluem todos os tipos gerados pelas atividades produtivas nas zonas rurais, quais sejam: resíduos agrícolas, florestais e pecuários. Os resíduos da pecuária são constituídos por estercos e outros produtos resultantes da atividade biológica dos bovinos, suínos, caprinos e outros, cuja relevância local justifica seu aproveitamento energético.

O biogás produzido através de digestão anaerobia é composto basicamente de metano $\left(\mathrm{CH}_{4}\right)$, dióxido de carbono $\left(\mathrm{CO}_{2}\right)$ e sulfeto de hidrogênio $\left(\mathrm{H}_{2} \mathrm{~S}\right)$. A digestão anaeróbia é um processo natural em que bactérias anaeróbias que atacam as estruturas da matéria orgânica para produzir compostos simples como metano, dióxido de carbono e água formam, assim, o biogás. O biogás contém aproximadamente 36 a $50 \%$ de metano $\left(\mathrm{CH}_{4}\right)$ e de 15 a $60 \%$ de dióxido de carbono $\left(\mathrm{CO}_{2}\right)$ (Rychebosh et al., 2011; Starr et al., 2012).

Resíduos de biomassa são considerados fonte de energia disponível embora possa causar grave degradação ambiental. A recuperação do biogás através da digestão anaeróbia é vista como forma ideal de tratamento de resíduos da biomassa. Diferentes tipos de resíduo têm potencial de produção de biogás tais como: dejetos de bovinos, de suínos, lodo de esgoto, resíduos de frutas e vegetais, entre outros (Qiao et al., 2011). Conforme a característica dos dejetos torna-se imprescindível o tratamento prévio dos resíduos suínos para depois aproveitá-los no solo. A biodigestão anaeróbia pode ser usada como tratamento, reduzindo o poder de poluição e gerando subprodutos como o biogás e o biofertilizante (Alvarez \& Gunnar, 2008).
Segundo Souza et al. (2004) uma outra vantagem no aproveitamento do biogás é o fato do metano ser um gás que contribui para o efeito estufa mais intensamente que o dióxido de carbono e sua queima para a geração de energia contribui sobremaneira para a redução de seu efeito como tal.

Diversos indicadores produtivos e econômicos da agropecuária levam para a grande produção de suínos, em especial na região sul. A aglomeração de unidades produtoras em determinada região e uma concentração maior de animais fazem com que surja um desafio que se pode agravar na suinocultura (Ahna et al., 2006). A produção de suínos traz problemas ambientais de forma mais intensa em certas regiões; no entanto, o manejo de dejetos apresenta pontos que podem trazer problemas para qualquer granja produtora (Orrico Júnior et al., 2009).

Sem tratamento, os dejetos poluem rios e emitam gases que aumentam o efeito estufa. O biogás proveniente da suinocultura vem-se tornando fonte de energia renovável. Além da produção de energia elétrica para suprir as necessidades dos produtores, contribui para a diminuição de danos ambientais; apesar disto, há aspectos desta produção que devem ser analisados cuidadosamente. Cita-se, aqui, a questão financeira da produção. A análise financeira de projetos de implantação de plantas de biogás é de fundamental importância visto que consiste em analisar os fatores econômicos essenciais para a obtenção de êxito no projeto, analisando o tempo de retorno do investimento.

Neste contexto objetivou-se, neste trabalho, mostrar a viabilidade ou não da produção de energia elétrica proveniente do biogás da suinocultura. Com cálculos de análise financeiros como VPL, TIR, tempo de retorno de investimento e custo de produção de energia elétrica, considerando-se a receita de venda de créditos carbono.

\section{Material e Métodos}

A Unidade da Granja Colombari localiza-se na Linha Marfim, no Município de São Miguel do Iguaçu, latitude $25^{\circ}$ 20' 53 Sul e longitude $54^{\circ} 14^{\prime}$ ' 16 Oeste, no oeste do estado do Paraná. A Granja Colombari trabalha com sistema de criação confinada. A geração de biomassa está diretamente ligada aos fatores de manejo, sistema de abastecimento de água, climatização e sistemas de limpeza. A propriedade possui dois biodigestores e um motor-gerador de $100 \mathrm{kVA}$ interligado à rede de distribuição de energia.

\section{Equações para determinação de parâmetros financeiros e do custo de produção da energia elétrica}

Foram estudados três cenários:

Cenário 1: Situação atual da Granja Colombari. A média de produção de biogás é de $554 \mathrm{~m}^{3} \mathrm{~d}^{-1}$ para uma média de 4673 suínos alojados a uma temperatura média de $22,11^{\circ} \mathrm{C}$; os dados do motor-gerador fornecido pelo fabricante são: potência - 76 $\mathrm{kW}$ e consumo de $50 \mathrm{~m}^{3} \mathrm{~h}^{-1}$ de biogás; os dados coletados do funcionamento do motor-gerador são de potência média de $66,22 \mathrm{~kW}$ e consumo específico $45,55 \mathrm{~m}^{3} \mathrm{~h}^{-1}$ de biogás. Para fins de cálculo serão usados dados reais de funcionamento. A geração de energia funciona durante $10 \mathrm{~h}$ diárias. Considera-se 
o custo do biodigestor de $\mathrm{R} \$ 294.600,00$ e do motor-gerador de R\$113.550,00.

Cenário 2: Simulando que a geração de energia funcione durante 16 h diárias; para isto, a produção de biogás precisará aumentar $729 \mathrm{~m}^{3} \mathrm{~d}^{-1} \mathrm{e}$, simultaneamente, a produção de suínos alcançará 6073 suínos. A potência e o consumo do motor serão considerados os mesmos nos três cenários. O custo do biodigestor será de $\mathrm{R} \$ 352.800,00$ e o custo do motor-gerador para termos de cálculo, será de R \$113.550,00.

Cenário 3: Simulando $20 \mathrm{~h}$ diárias de geração de energia, sendo $911 \mathrm{~m}^{3} \mathrm{~d}^{-1}$ de biogás e uma produção de suínos de 7000 animais, considerar-se-á o custo do biodigestor de R $\$$ $441.000,00$ e do motor-gerador, de $\mathrm{R} \$ 113.550,00$.

Os valores comerciais do biodigestor (incluindo escavação, instalação completa) foram fornecidos pela empresa Sansuy Soluções em PVC e os do motor-gerador, pela empresa ER-BR Energias Renováveis.

Nos três cenários foi calculado o custo da produção de energia elétrica; jáos parâmetros de avaliação econômica de projetos serão expostos através VPL, TIR, TRI.

Valor presente líquido (VPL) $\rightarrow$ faz uma comparação do investimento realizado com o valor presente nos fluxos de caixa gerados pelo projeto; leva em conta todos os fluxos de caixa e não apenas o instante no tempo em que o saldo acumulado se torna positivo, que pode nos dar uma medida de riqueza adicionada (VPL $>0$ ) ou destruída (VPL $<0)$. A taxa de juros a ser utilizada para definição dos parâmetros será de 5,5\% a.a conforme imposto pelo Programa ABC. VPL é dado por:

$$
\mathrm{VPL}=-1+\sum_{\mathrm{t}=1}^{\mathrm{n}} \frac{\mathrm{FC}_{\mathrm{t}}}{(1+\mathrm{r})^{\mathrm{t}}}+\frac{\mathrm{VR}}{(1+\mathrm{r})^{\mathrm{n}}}
$$

donde:

I - investimento inicial

$\mathrm{FC}_{\mathrm{t}}$ - fluxo de caixa líquido na data $\mathrm{t}$

$r \quad$ - custo de capital

VR - valor residual do projeto ao final do período de análise

Taxa Interna de Retorno (TIR) $\rightarrow$ É uma referência a ser utilizada para definir a aceitação ou não de um projeto. A taxa interna de retorno torna o VPL nulo.

Tempo de retorno de investimento (TRI) $\rightarrow$ para o cálculo de TRI o valor pago ao produtor pelo MWh produzido foi simulado em $R \$ 120,00-R \$ 140,00-R \$ 160,00-R \$ 180,00$ - R\$200,00 - R \$220,00 porque, conforme ANEEL (2010) o valor pago pela energia provinda da biomassa é de R \$ 144,20 o MWh. Para análise de viabilidade o valor considerado será de R\$ 140,00 o MWh.

$$
\mathrm{TRI}=\frac{\ln \left(\frac{\mathrm{k}}{\mathrm{k} \times(1-\mathrm{j})^{-1}-\mathrm{j}}\right)}{\ln (1+\mathrm{j})}
$$

donde:

$\mathrm{k} \quad-(\mathrm{A} / \mathrm{CI})-(\mathrm{OM} / 100)$

CI - custo de investimento no sistema biodigestor/motorgerador, R\$
A - gasto anual com energia elétrica adquirida na rede, $\mathrm{R} \$ \mathrm{ano}^{-1}$

$\mathrm{OM}$ - gastos com amortização e manutenção da planta, $\mathrm{R} \$ \mathrm{ano}^{-1}$

TRI - tempo de retorno, anos

Custo da energia elétrica:

$$
\mathrm{CE}=\frac{\mathrm{CAG}+\mathrm{CAB}}{\mathrm{PE}}
$$

em que:

$\mathrm{C}^{\mathrm{E}} \quad$ - custo de energia elétrica produzida via biogás, $\mathrm{R} \$ \mathrm{kWh}$

$\mathrm{CAB}$ - gasto anual com biogás, $\mathrm{R} \$ \mathrm{ano}^{-1}$

PE - produção de eletricidade pela planta de biogás, $\mathrm{kWh}$ ano $^{-1}$

CAG - custo anualizado do investimento no conjunto motorgerador, $\mathrm{R} \$ \mathrm{ano}^{-1}$

$$
\begin{gathered}
\mathrm{CAG}=\mathrm{CIG} \times \mathrm{FRC}+\frac{\mathrm{CIG} \times \mathrm{OM}}{100} \\
\mathrm{CAB}=\mathrm{CB} \times \mathrm{CNB}
\end{gathered}
$$

em que:

CIG - custo do investimento no motor-gerador, R\$

$\mathrm{OM}$ - custo com organização e manutenção, $\%$ ano $^{-1}$

$\mathrm{CB}$ - custo do biogás, $\mathrm{R} \$ \mathrm{~m}^{-3}$

CNB - consumo de biogás pelo conjunto motor-gerador, $\mathrm{m}^{3} \mathrm{ano}^{-1}$

A produção de eletricidade (PE) é dada por:

$$
\mathrm{PE}=\mathrm{Pot} \times \mathrm{T}
$$

em que:

Pot - potência nominal da planta, $\mathrm{kW}$

$\mathrm{T}$ - disponibilidade anual da planta, $\mathrm{h} \mathrm{ano}^{-1}$

O fator de recuperação de capital é dado por:

$$
F R C=\frac{j \times(1+j)^{n}}{(1+j)^{n-1}-1}
$$

em que:

FRC - fator de recuperação de capital

j - taxa de desconto, $\%$ ano

n - anos para amortização do investimento

O custo do biogás é dado por:

$$
\mathrm{CB}=\frac{\mathrm{CAB}}{\mathrm{PAB}}
$$

em que:

$\mathrm{CAB}$ - custo anualizado do investimento no biodigestor, $\mathrm{R} \$$ ano $^{-1}$

$\mathrm{PAB}$ - produção anual de biogás, $\mathrm{m}^{3}$ ano $^{-1}$ 


$$
\mathrm{CAB}=\mathrm{CIB} \times \mathrm{FRC}+\frac{\mathrm{CIB} \times \mathrm{OM}}{100}-\mathrm{CC}
$$

em que:

CIB - custo de investimento no biodigestor, $\mathrm{R} \$$

$\mathrm{PAB}$ - produção anual de biogás, $\mathrm{m}^{3} \mathrm{ano}^{-1}$

$\mathrm{CC}$ - ganho com crédito carbono, $\mathrm{R} \$ \mathrm{ano}^{-1}$

Para conversão da quantidade de metano em crédito carbono, foi usada a Eq.10.

- Conversão de biogás em t $\mathrm{CO}_{2}$ e convertido em $\mathrm{R} \$ \mathrm{t}^{-1}$

Para tal conversão foram usados os seguintes parâmetros:

Produção de biogás em $\mathrm{m}^{3} \mathrm{ano}^{-1}, \mathrm{Pr}$

Densidade $\mathrm{CH}_{4}: 0,35 \mathrm{~m}^{3} \mathrm{CH}_{4} \mathrm{~kg}^{-1}$, Ds

Porcentagem de metano no biogás: $60 \%$, Pc

Valor por $\mathrm{tCO}_{2}$ : U\$ 14,50 .

Equivalência entre $\mathrm{CH}_{4}$ e $\mathrm{CO}_{2}-1$ unidade de $\mathrm{CH}_{4}$ equivale a 21 unidade de $\mathrm{CO}_{2}(\mathrm{Un})$

$$
\mathrm{tCO}_{2}=\frac{\operatorname{Pr} \times \mathrm{Ds} \times \mathrm{Pc} \times \mathrm{Un}}{1000}
$$

valor da tonelada $=\mathrm{tCO}_{2} \times \mathrm{US}$

Para o cálculo do beneficio da produção de biofertilizante foi usada a metodologia de Cervi (2009).

$$
\mathrm{BPB}=\mathrm{QN} \times \mathrm{PNM}
$$

donde:

$\mathrm{BPB}$ - benefício de produção de biofertilizante, $\mathrm{R} \$ \mathrm{ano}^{-1}$

QN - wuantidade de nutrientes presentes, $\mathrm{kg}_{\mathrm{ano}}{ }^{-1}$

PNM - preço comercial dos nutrientes no mercado, $\mathrm{R} \$ \mathrm{~kg}^{-1}$

O biofertilizante de suínos contém $2,15 \%$ de nitrogênio $(\mathrm{N})$, $1,6 \%$ de fosfato $\left(\mathrm{P}_{2} \mathrm{O}_{5}\right)$ e $1,15 \%$ de óxido de potássio $\left(\mathrm{K}_{2} \mathrm{O}\right)$ (Tanganelli, 2009).

\section{Resultados E Discussão}

A Figura 1 representa a variação do tempo de retorno do investimento em função das horas de produção de energia e do valor pago pela concessionária de energia. Com produção de $10 \mathrm{~h} \mathrm{~d}^{-1}$ sem a venda de crédito carbono $\mathrm{R} \$ 140,00 \mathrm{o}$ $\mathrm{MWh}$, o tempo de retorno do investimento gira em torno de 25 anos. Com a receita da venda de crédito carbono, o TRI cai para 10 anos.

Com a produção em $16 \mathrm{~h} \mathrm{~d}^{-1}$, sem a venda de crédito carbono o TRI está na faixa de 12 anos. Com a venda de crédito carbono o tempo de retorno de investimento é de 8 anos.

A Tabela 1 mostra a variação do custo da energia elétrica de acordo com a venda ou não de crédito carbono. Com a produção de energia em $10 \mathrm{~h} \mathrm{~d}^{-1}$ sem a venda de crédito carbono, com o tempo de retorno do investimento estimado em 10,15 ou 20 anos, o valor da produção de energia, R \$289,94, R \$219,50 e R\$ 185,80 , respectivamente, é superior ao pago pela concessionária de energia que é R\$140,00 o MWh.
A. $10 \mathrm{~h} \mathrm{dia}^{-1}$

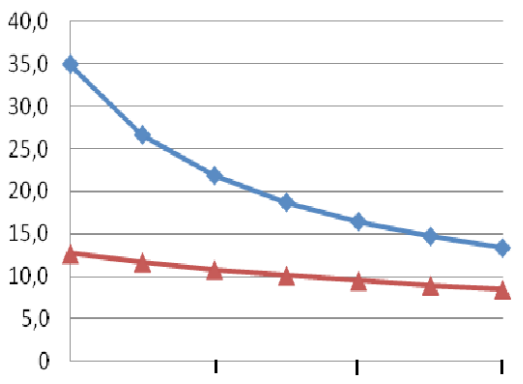

B. $16 \mathrm{~h} \mathrm{dia}^{-1}$

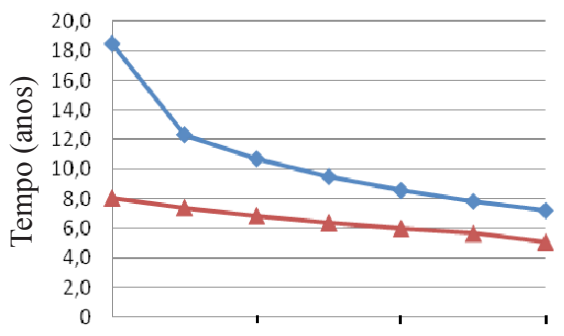

C. $20 \mathrm{~h} \mathrm{dia}^{-1}$

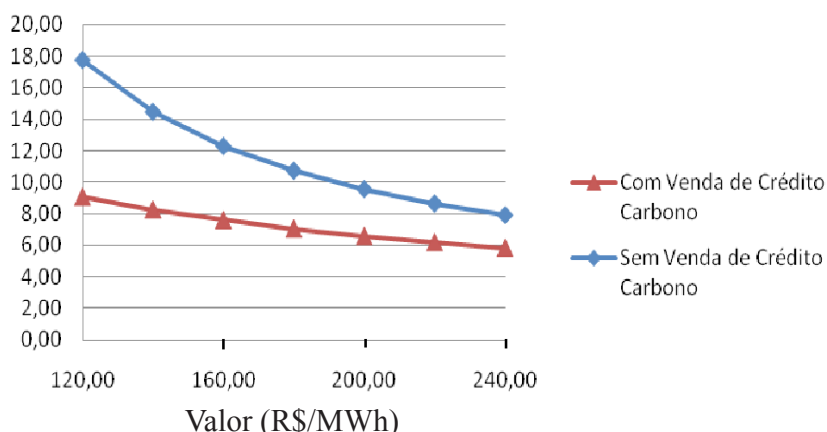

Figura 1. Tempo de retorno de investimento

Tabela 1. Custo da energia elétrica ( $R \$ M W h)$ proveniente do biogás da suinocultura variando o tempo de produção em função do tempo de retorno de investimento

\begin{tabular}{ccc}
\hline Tempo de & \multicolumn{2}{c}{$\mathbf{1 0 ~ \mathbf { ~ d } ^ { - 1 }}$} \\
\cline { 2 - 3 } $\begin{array}{c}\text { retorno de } \\
\text { investimento (anos) }\end{array}$ & $\begin{array}{c}\text { Sem venda de } \\
\text { crédito carbono (R\$) }\end{array}$ & $\begin{array}{c}\text { Com a venda de } \\
\text { crédito carbono (R\$) }\end{array}$ \\
10 & 289,94 & 196,40 \\
15 & 219,50 & 126,11 \\
20 & 185,80 & 92,32 \\
\hline
\end{tabular}

A Tabela 2 mostra que, com $16 \mathrm{~h} \mathrm{~d}^{-1}$, o valor fica competitivo a partir do tempo de retorno igual 20 anos quando o valor da produção de energia chega a R\$132,60 MWh. Considerando a venda de crédito carbono com 10 anos, torna-se viável o custo da produção de energia elétrica, que fica em torno de R \$130,20,

Tabela 2. Custo da energia elétrica ( $\mathrm{R} \$ \mathrm{MWh}$ ) proveniente do biogás da suinocultura variando o tempo de produção em função do tempo de retorno de investimento

\begin{tabular}{ccc}
\hline \multirow{2}{*}{$\begin{array}{c}\text { Tempo de } \\
\text { retorno de }\end{array}$} & \multicolumn{2}{c}{$\mathbf{1 6} \mathbf{~ h ~ d}^{-\mathbf{1}}$} \\
\cline { 2 - 3 } investimento (anos) & $\begin{array}{c}\text { Sem venda de } \\
\text { crédito carbono (R\$) }\end{array}$ & $\begin{array}{c}\text { Com a venda de } \\
\text { crédito carbono (R\$) }\end{array}$ \\
10 & 207,10 & 130,20 \\
15 & 156,70 & 79,90 \\
20 & 132,60 & 55,77 \\
\hline
\end{tabular}


gerando renda extra ao produtor com a venda do excedente de energia à concessionária.

Oliveira \& Martins (2007) estudaram a viabilidade econômica do uso de um gerador disponibilizando $40 \mathrm{kWh}$, utilizando biogás da suinocultura e concluíram que esta alternativa é viável sempre a demanda e o preço da energia aumentam. Com uma tarifa de R \$200,00 MWh, o tempo retorno do investimento, considerando-se desconto da taxa de juros, foi de 39; 26 e 19 meses para tempos de geração diária de 10; 14 e $18 \mathrm{~h}$, respectivamente.

Quando a produção de energia é de $20 \mathrm{~h} \mathrm{~d}^{-1}$, o TRI é, sem a venda de crédito carbono, de 14 anos; com a venda de crédito carbono, cde 8 anos.

$\mathrm{Na}$ Tabela 3 constam os custos de produção de energia elétrica operando $20 \mathrm{~h} \mathrm{~d}^{-1}$, variando o tempo de retorno de investimento. Constata-se que sem a venda de crédito carbono o retorno do investimento ocorre em 20 anos com preço competitivo da energia; já com a venda de crédito carbono com 10 anos o investimento tem retorno, ainda gerando renda ao produtor com a venda do excedente de energia.

Tabela 3. Custo da energia elétrica ( $R$ \$ MWh) proveniente do biogás da suinocultura com o tempo de produção em função do tempo de retorno de investimento

\begin{tabular}{ccc}
\hline Tempo de & \multicolumn{2}{c}{$20 \mathbf{h ~ d}^{-1}$} \\
\cline { 2 - 3 } $\begin{array}{c}\text { retorno de } \\
\text { investimento (anos) }\end{array}$ & $\begin{array}{c}\text { Sem venda de } \\
\text { crédito carbono (R\$) }\end{array}$ & $\begin{array}{c}\text { Com a venda de } \\
\text { crédito carbono (R\$) }\end{array}$ \\
10 & 197,02 & 120,11 \\
15 & 149,10 & 72,26 \\
20 & 126,22 & 49,31 \\
\hline
\end{tabular}

Segundo Zago (2003) o empreendimento passa a ser economicamente viável quando a propriedade possui capacidade de produção de $200 \mathrm{~m}^{3} \mathrm{~d}^{-1}$ de gás, o que gera uma produção aproximada de $300 \mathrm{kVA} \mathrm{h} \mathrm{d}^{-1}$. Para Stegelin (2010) os benefícios de energia gerada pelo biogás consistem em economia no custo da energia elétrica comprada, economia com combustível quando o biogás é usado para esse fim; economia com a compra de gás natural para aquecimento de água e outras instalações; evita-se o custo com fertilizantes comerciais usando o esterco bruto para fertilização e mais a receita de crédito carbono nos mercados de gases de efeito estufa.

Yiridoe et al. (2009) e Bronw et al. (2007) constataram, estudando a viabilidade de produção de biogás para geração de energia elétrica em laticínios, que não era viável (US\$ 0,12 $\mathrm{kWh}$ ) a produção de eletricidade em laticínios de pequeno porte. Para produtores pequenos de suínos também se tornou inviável a produção de biogás, ficando no mesmo patamar dos pequenos laticínios (US\$ 0,12 kWh). Gwavuya et al. (2012) encontraram, estudando o uso do biogás em domicílios da Etiópia, taxa de retorno próximo dos $10 \%$ com planta de biogás com capacidade de $6 \mathrm{~m}^{3}$, usando dejetos de animais.

Coldebella et al. (2006) constataram, analisando a produção de energia elétrica via biogás da bovinocultura, que o custo de produção de energia elétrica está diretamente associado ao tempo de amortização do investimento e de operação do sistema. Com uma produção de $10 \mathrm{~h} \mathrm{~d}^{-1}$ num tempo de armotização de 10 anos, o valor da produção (R\$ 183,69) de energia foi menor que os dos resultados obtidos.

Brasil (2012) relata que as fontes renovaveis de energia elétrica tendem a aumentar significativamente em 5 anos, visto que o custo de implantação de tais fontes está diminuindo. No leilão realizado pelo governo federal visando à compra de energia eólica, o megawatt-hora foi negociado a R\$ 180,00 mas a energia solar não está em um patamar competivo, o preço do megawatt-hora gira entre $\mathrm{R} \$ 300,00$ e $\mathrm{R} \$ 400,00$. O custo elevado se deve ao alto investimento inicial para a produção de energia solar.

Walla \& Schneeberger (2005) constataram, ao analisar a viabilidade de usinas de biogás, uma mistura de variáveis relevantes: a eficiência econômica da digestão anaeróbia, entre outros, como o custo de investimento, custo de exploração da unidade de biogás ea produção de metano .

Com a venda de crédito carbono o valor da produção de energia reduz drasticamente;com tempo de retorno de 10 , 15 e 20 anos gerando 10,16 e $20 \mathrm{~h} \mathrm{~d}^{-1}$, há uma redução de custos de aproximadamente $60 \%$, com a venda de crédito carbono;produzindo 10, 16 e $20 \mathrm{~h}$, ganha-se com crédito carbono R\$22.587,00 - R\$ 29.735,06 - e R\$37.168,83, respectivamente.

A Tabela 4 mostra o custo de produção de energia considerando o investimento do biodigestor $30 \%$ do valor total, e apenas a instalação da vinimanta, sem a venda de crédito carbono. Em 15 anos e $10 \mathrm{~h} \mathrm{~d}^{-1}$, o custo da produção de energia elétrica (R\$108,64 MWh) torna-se viável ao produtor; nas outras condições expostas na Tabela 3, todas mostram viabilidade na produção de energia elétrica via biogás da suinocultura.

Com relação ao beneficio do biofertilizante, os resultados estão demonstrados na Tabela 5 .

Observa-se, conforme o número de suínos, que a quantidade de dejetos e, em contrapartida, o biofertilizante aumenta. Com 4673, 6073 e 7000 suínos, o produtor deixa de gastar com

Tabela 4. Custo da energia elétrica ( $\left.R \$ M W h^{-1}\right)$ proveniente do biogás da suinocultura variando o tempo de produção em função do tempo de retorno de investimento, com investimento de $30 \%$ do total do valor do biodigestor

\begin{tabular}{cccc}
\hline \multirow{2}{*}{$\begin{array}{c}\text { Tempo de } \\
\text { retorno (anos) }\end{array}$} & \multicolumn{3}{c}{$\begin{array}{c}\text { Tempo de produção de energia } \mathbf{h} \mathbf{d}^{-1} \\
\text { sem venda de crédito carbono (R\$) }\end{array}$} \\
\cline { 2 - 4 } 10 & $\mathbf{1 0}$ & $\mathbf{1 6}$ & $\mathbf{2 0}$ \\
15 & 143,45 & 97,43 & 87,34 \\
20 & 108,64 & 73,74 & 66,13 \\
& 91,92 & 62,42 & 55,96 \\
\hline
\end{tabular}

Tabela 5. Beneficio do biofertilizante em relação ao número de suínos

\begin{tabular}{|c|c|c|c|}
\hline $\mathrm{N}^{0}$ de suínos & 4673 & 6073 & 7000 \\
\hline $\begin{array}{l}\text { Quantidade de biofertilizante } \\
\text { produzido (t ano-1) }\end{array}$ & $1.998,5$ & $2.597,3$ & $2.993,7$ \\
\hline Nitrogênio (R\$ ano-1) & $\mathrm{R} \$ 56.718,81$ & $\mathrm{R} \$ 73.711,40$ & $\mathrm{R} \$ 8$ \\
\hline Fosfato ( $\mathrm{RS}$ ano-1) & $\mathrm{R} \$ 44.767,49$ & $\mathrm{R} \$ 58.179,53$ & $\mathrm{R} \$ 67.060,22$ \\
\hline $\begin{array}{l}\text { Oxido de Potássio } \\
\left(\mathrm{R} \$ \text { ano }^{-1}\right)\end{array}$ & $\mathrm{R} \$ 44.367,78$ & $\mathrm{R} \$ 58.179,53$ & $\mathrm{R} \$ 66.461,47$ \\
\hline Total & $\mathrm{R} \$ 145.854$, & $190.070,4$ & $\$ 218.484,59$ \\
\hline
\end{tabular}


fertilizantes convencionais $\mathrm{R} \$ 145.854,08, \mathrm{R} \$ 190.070,46, \mathrm{R} \$$ 218.484,59 por ano, respectivamente. Este valor tem relação direta com a quantidade de nutrientes do biofertilizante.

Com relação ao VPL, a Figura 2 mostra os valores em quanto ao tempo de retorno do investimento e operação do motor-gerador, sem a venda de crédito carbono.

Na situação em que o VPL é negativo (10 anos por $\left.10 \mathrm{~h} \mathrm{~d}^{-1}\right)$ a implantação do sistema de geração de energia elétrica não é viável a 5,5\% a.a., o que resultaria em um prejuízo, ao produtor, de $\mathrm{R} \$ 8.519,21$.

A Figura 2B mostra o VPL negativo em várias situações considerando-se o valor total do investimento. O VPL se torna

A.

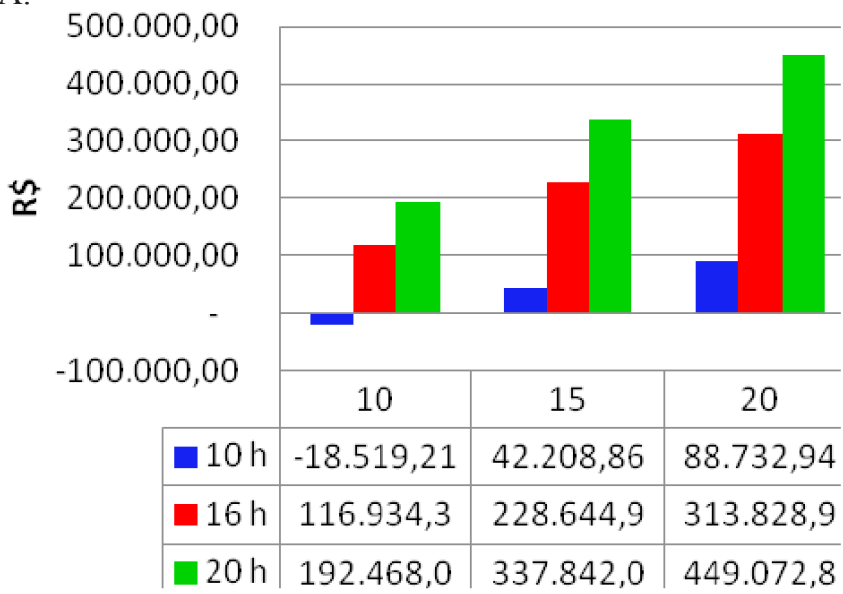

B.

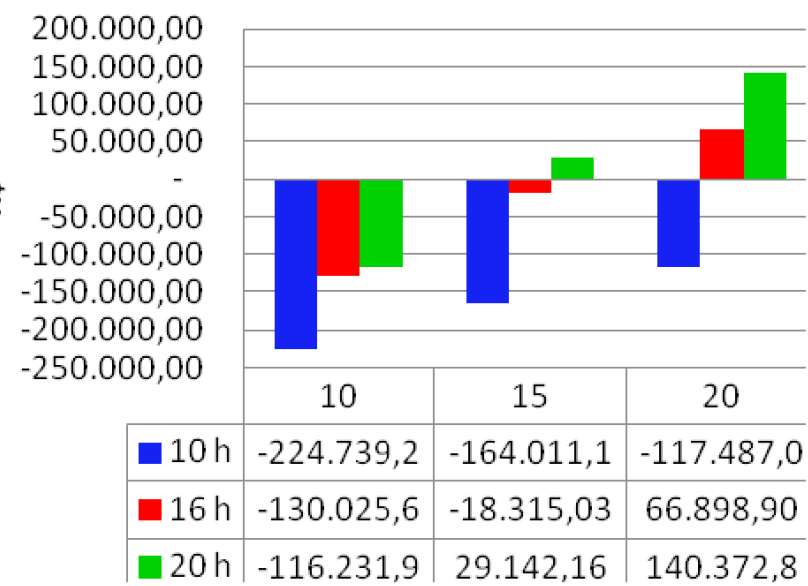

C.

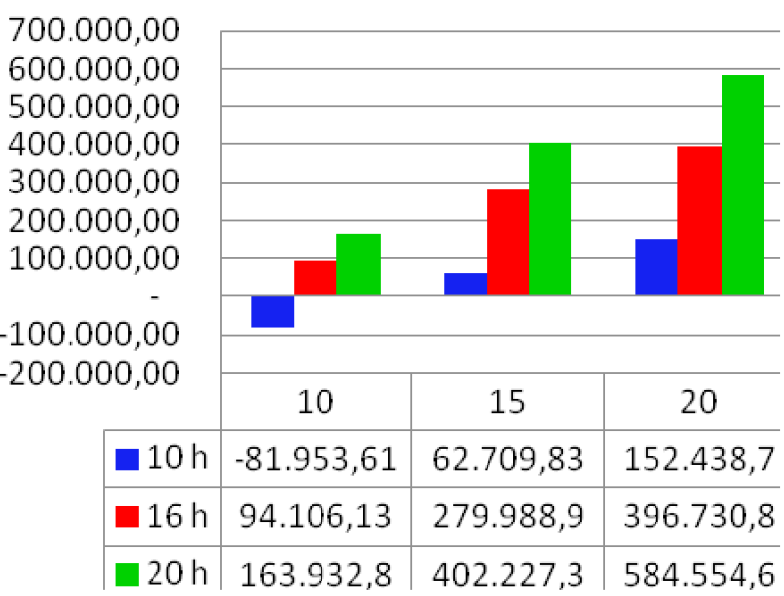

Figura 2. Valor presente líquido positivo a partir da situação de 15 anos de TRI operando $20 \mathrm{~h}$ $\mathrm{d}^{-1}$. No entanto, o VPL volta a ser negativo quando opera em 10 $\mathrm{h} \mathrm{d}^{-1}$ com 20 anos de TRI; a partir daí, o VPL se torna positivo novamente, ou seja, na maior parte das situações analisadas nessas condições estudadas, o produtor terá prejuízo se produzir energia proveniente do biogás da suinocultura. Gwavuya et al. (2012) constataram, analisando a coleta de esterco para produção de biogás e energia para residências, que no tempo de retorno de 20 anos o VPL foi positivo, com base em uma taxa de desconto de $4 \%$.

A Figura 2C representa o VPL em função do tempo de retorno considerando o valor total do investimento com a venda de crédito carbono.

Quando há renda com a venda de crédito carbono, há apenas uma situação que gera prejuízo ao produtor. Quando se geram $10 \mathrm{~h} \mathrm{~d}^{-1}$ e se tem um tempo de amortização de 10 anos. Se o produtor insistir no projeto de geração de eletricidade sofrerá um prejuízo na casa dos $\mathrm{R} \$ 81.953,61$; as outras situações geram uma margem razoável de lucro; isto ocorre porque o mercado de carbono é uma área em expansão em todo o planeta, pelo fato de contribuir com o meio ambiente e, ao mesmo tempo, gerar renda a pequenos e grandes produtores de alimentos; ele não contempla apenas a criação de animais mas todas as fontes de energia renovável, de forma geral.

Devuyst et al. (2011) concluíram, estudando a diferença entre a produção de energia alternativa, comparando etanol, biogás e esterco de gado que, nos dois cenários, a taxa de juros variou, o primeiro com taxa de $4,5 \%$ e o segundo com taxa de 6\%. Em ambos, o VPL calculado esteve negativo, chegando-se a conclusão que, mesmo o VPL sendo negativo, ainda é viável a instalação de biodigestor visando à produção e à venda de energia elétrica excedente, do que apenas para tratamento de resíduos agrícolas.

Kunz et al. (2009) constataram, estudando sistema de tratamento de dejetos de suínos, que com a venda de crédito carbono ocorre diminuição no custo de produção, de 14 a 18\%; quando o sistema de tratamento de dejetos é instalado em propriedades rurais tem potencial para gerar receitas adicionais com biogás e biofertilizantes; essas receitas podem representar um valor presente líquido positivo, com uma taxa interna de retorno (TIR) variando de 6,4 para $28,4 \%$ por ano dependendo das metas ambientais e da escala do projeto.

\section{Conclusões}

1. Na situação atual da propriedade, variando o tempo de retorno não há competitividade para a produção de energia elétrica.

2. Quando há venda de crédito carbono o custo de produção de energia cai em média $60 \%$.

3. Aumentando a produção de biogás, o custo da energia elétrica tem redução.

4. O VPL torna-se positivo quando o tempo de retorno do investimento é de 15 anos e produção a de $20 \mathrm{hd}^{-1}$ sem a venda de crédito carbono.

5. Com a venda de crédito carbono o VPL se torna positivo com um tempo de retorno de 10 anos e produção diária de 16 $\mathrm{h} \mathrm{d}^{-1}$. 
6. O produtor deixa de gastar por ano, com fertilizantes convencionais, aproximadamente R\$ $145.854,00$; é uma forma, portanto, de diminuir gastos e aumentar os lucros com a produção de grãos e pastagens.

\section{Literatura Citada}

Ahna, J.; Doa, T. H.; Kimb, S. D.; Hwang, S. The effect of calcium on the anaerobic digestion treating swine wastewater. Biochemical Engineering Journal, v.30, p.3338, 2006.

Alvarez, R.; Gunnar, L. Semi-continuous co-digestion of solid slaughterhouse waste, manure, and fruit and vegetable waste. Renewable Energy, v.33, p.726-734, 2008.

ANEEL - Agência Nacional de Energia Elétrica. Atlas da Energia Elétrica no Brasil. 2010.

BEN - Balanço Energético Nacional 2011: Ano base 2010/ Empresa de Pesquisa Energética. Rio de Janeiro: EPE, 2011. < https://ben.epe.gov.br/downloads/Relatorio_final_ BEN_2011.pdf>. 23 Mar. 2012.

Brasil - Portal Brasil. <http://www.brasil.gov.br>. 12 Jun. 2012.

Brown B. B.; Yiridoe E. K.; Gordon R. Impact of single versus multiple policy options on the economic feasibility of biogás energy production: Swine and dairy operations in Nova Scotia. Energy Policy, v.35, p.4597-4610, 2007.

Cervi, R. G. Avaliação econômica do aproveitamento de biogás e do biofertilizante produzidos por digestão anaeróbia. Botucatu: UNESP. 2009. 57p. Dissertação Mestrado

Coldebella, A. Souza, S. N. M. de; Souza, J. de; Kohele, A. C. Viabilidade da cogeração de energia elétrica com biogás da bovinocultura de leite. Encontro de Energia no Meio Rural, v.2, p.1-9, 2006.

Devuyst, E. A.; Pryor, S. W.; Lardy, G.; Eide, W.; Wiederholt, R. Cattle, ethanol, and biogás: Does closing the loop make economic sense? Agricultural Systems, v.104, p.609-614. 2011.

Gwavuya, S. G.; Abele S.; Barfuss, I.; Zeller M., Müller, J. Household energy economics in rural Ethiopia: A costbenefit analysis of biogás energy. Renewable Energy, v.48, p.202-20, 2012.

IBGE - Instituto Brasileiro de Geografia e Estatística. Censo agropecuário. 2006. 777p.
Kunz, A.; Miele, M.; Steinmetz R. L. R. Advanced swine manure treatment and utilization in Brazil. Bioresource Technology, v.100, p.5485-5489, 2009.

Oliveira, P.A.V. de; Martins, F.M. Utilização do biogás na suinocultura para geração de energia elétrica. In: Congresso Brasileiro de Engenharia Agrícola, 36, 2007, Bonito. Anais...Jaboticabal: SBEA, 2007. CD-Rom

Orrico Júnior, M. A. P.; Orrico A. C. A. Lucas Júnior, J. de. Biodigestão anaeróbia de dejetos de suínos com e sem separação da fração sólida em diferentes tempos de retenção hidráulica. Engenharia Agrícola, v.29, p.474-482. 2009.

Quiao, W.; Yan, X.; Ye, J.; Sun, Y.; Wang, W.; Zhang, Z. Evolution of biogás production from different biomass wastes with/without hydrothermal pretreatment. Renewable Energy, v.36, p.3313-3318, 2011.

Ryckebosch, E.; Drouilan, M.; Vervaeren, H. Techniques for transformation of biogás to biomethane. Biomass e Bioenergy, v.35, p.1633-1645, 2011.

Souza, S. N. M. de; Pereira, W. C.; Pavan, A. A. Custo da eletricidade gerada em conjunto motor gerador utilizando biogás da suinocultura. Acta Scientiarum Technology, v.26, p.127-133, 2004.

Starr, K.; Gabarrel, X.; Villalba, G.; Talens, L.; Lombardi L. Life cycle assessment of biogásupgrading technologies. Waste Management, v.32, p.991-999, 2012.

Stelegin, F. Sustainable economic, marketing, environmental and financial opportunities for biogas recovery systems. In: WERA-72 Agribusiness Research Emphasizing Competitiveness and Profitability Meeting, Santa Clara, 2010. Anais... Santa Clara: WERA, 2010. CD-Rom

Tanganelli, K. M. Utilização dos dejetos de suínos como fertilizante do solo - Oklahoma State University. Botucatu: Universidade Estadual Paulista, 2007. 21p. Relatório de Estágio Curricular

Walla, C.; Schneeberger, W. The optimal size for biogás plants. Biomass and Bioenergy, v.32, p.551-557, 2009.

Yiridoe, E.; Gordon, R.; Brown, B. Nonmarket cobenefits and economic feasibility of on-farm biogás energy production. Energy Policy, v.37, p.1170-1179, 2009.

Zago, S. Potencialidade de produção de energia através do biogás integrada à melhoria ambiental em propriedades rurais com criação intensiva de animais, na região do meio oeste catarinense. Blumenau: URB, 2003. 103p. Dissertação Mestrado 